\title{
Uptake of a fluorescent L-glucose derivative 2-NBDLG into three-dimensionally accumulating insulinoma cells in a phloretin-sensitive manner
}

\author{
Ayako Sasaki $^{1} \cdot$ Katsuhiro Nagatomo $^{1} \cdot$ Koki Ono $^{1} \cdot$ Toshihiro Yamamoto $^{2,3} \cdot$ \\ Yuji Otsuka $^{2} \cdot$ Tadashi Teshima $^{2} \cdot$ Katsuya Yamada $^{1}$
}

Received: 29 September 2015/Accepted: 10 October 2015/Published online: 9 November 2015

(c) The Author(s) 2015. This article is published with open access at Springerlink.com

\begin{abstract}
Of two stereoisomers of glucose, only D- and not L-glucose is abundantly found in nature, being utilized as an essential fuel by most organisms. The uptake of Dglucose into mammalian cells occurs through glucose transporters such as GLUTs, and this process has been effectively monitored by a fluorescent D-glucose derivative 2-[N-(7-Nitrobenz-2-oxa-1,3-diazol-4-yl)amino]-2-deoxyD-glucose (2-NBDG) at the single cell level. However, since fluorescence is an arbitrary measure, we have developed a fluorescent analog of L-glucose 2-[N-(7Nitrobenz-2-oxa-1,3-diazol-4-yl)amino]-2-deoxy-L-glucose (2-NBDLG), as a negative control substrate for more accurately identifying the stereoselectivity of the uptake. Interestingly, a small portion of mouse insulinoma cells MIN6 abundantly took up 2-NBDLG at a late culture stage ( $\gtrsim 10$ days in vitro, DIV) when multi-cellular spheroids exhibiting heterogeneous nuclei were formed, whereas no such uptake was detected at an early culture stage $(\lesssim 6$ DIV). The 2-NBDLG uptake was persistently observed in the presence of a GLUT inhibitor cytochalasin B. Neither D- nor L-glucose in $50 \mathrm{mM}$ abolished the uptake. No
\end{abstract}

Electronic supplementary material The online version of this article (doi:10.1007/s13577-015-0125-3) contains supplementary material, which is available to authorized users.

Katsuya Yamada

kyamada@hirosaki-u.ac.jp

1 Department of Physiology, Hirosaki University Graduate School of Medicine, 5 Zaifu-cho, Hirosaki,

Aomori 036-8562, Japan

2 Peptide Institute, Inc., Saito Research Center, Ibaraki, Osaka 567-0085, Japan

3 Graduate School of Science, Osaka University, Toyonaka, Osaka 560-0043, Japan significant inhibition was detected by inactivating sodium/ glucose cotransporters (SGLTs) with $\mathrm{Na}^{+}$-free condition. To our surprise, the 2-NBDLG uptake was totally inhibited by phloretin, a broad spectrum inhibitor against transporters/channels including GLUTs and aquaporins. From these, a question might be raised if non-GLUT/non-SGLT pathways participate in the 2-NBDLG uptake into spheroid-forming MIN6 insulinoma. It might also be worthwhile investigating whether 2-NBDLG can be used as a functional probe for detecting cancer, since the nuclear heterogeneity is among critical features of malignancy.

Keywords L-Glucose Tumor - Spheroid - Cancer · Biomarker

\section{Introduction}

Mammalian cells take up D-glucose in a stereoselective manner through plasma membrane transporters, such as GLUTs, whereby only D- and not L-glucose is recognized [1]. We have shown that 2-[N-(7-Nitrobenz-2-oxa-1,3-diazol-4-yl)amino]-2-deoxy-D-glucose (2-NBDG) (Online Resource 1a), which was originally synthesized to see the viability of E. coli cells [2], is taken up into mammalian cells through GLUTs in a time, concentration and temperature-dependent manner [3, 4]. So far, 2-NBDG has been effectively utilized for monitoring D-glucose uptake in a variety of mammalian cells including pancreatic cells $[3$, 5], brain cells [6-9], and tumor cells [10-15]. However, since fluorescence is an arbitrary measure, a control substrate has been awaited for more accurately evaluating the GLUT-mediated component [4].

A green fluorescence-emitting 2-[N-(7-Nitrobenz-2-oxa1,3-diazol-4-yl)amino]-2-deoxy-L-glucose (2-NBDLG), the 
mirror-image isomer of 2-NBDG, was thus developed as the control substrate (Online Resource 1b) [16, 17]. For evaluating an occurrence of non-specific uptake such as due to a loss of membrane integrity, we found it strongly helpful to use 2-NBDLG simultaneously with a membrane-impermeable L-glucose derivative 2-TRLG, which bears a large red fluorophore Texas red (Online Resource 1c) [17].

To explore the stereoselective uptake of mammalian cells with such fluorescent tracers, we have used mouse insulin-secreting clonal (MIN6) cells [18]. Surprisingly, when MIN6 cells cultured over 10 days in vitro (DIV) were examined, the fluorescence of cells increased significantly not only by application of 2-NBDG, but also by 2-NBDLG. In the present study, we characterize unique features of the 2-NBDLG uptake into the insulinoma cells.

\section{Methods}

\section{Confocal microscopic study}

\section{Culture}

MIN6 cells were grown, according to the original protocol in Dulbecco's modified Eagle's medium (DMEM) (D5648, Sigma-Aldrich) [18]. Only cells in earlier passages (from 5 up to 10 times) were used in the present study except for Online Resource 2 [19]. On the day of culture, poly-L-lysine hydrobromide (PLL) (P6282, final concentration 1/500, Sigma)-coated, small glass coverslips (No. 0, Matsunami) were placed on $35 \mathrm{~mm}$ non-coated dish (Iwaki). Cells for the confocal measurement were seeded at a density of 1000 cells per cover slip. Culture medium was half exchanged every 3 days.

\section{Measurement}

The tracer application and image acquisition were conducted by modifying the method reported previously [4, 17]. In brief, a temperature-controlled custom made chamber was placed on a motor-driven xyz stage of a laser confocal microscope (TCS-SP5, Leica). 2-NBDG/2NBDLG and 2-TRLG were excited by a single $488 \mathrm{~nm}$ laser source and the fluorescence was detected in 500-580 $\mathrm{nm}$ and 580-740 $\mathrm{nm}$ wavelength range, respectively, with a dichroic mirror at $500 \mathrm{~nm}$ (RSP 500). 4',6diamidino-2-phenylindole (DAPI) was applied for nuclear staining in live-cell condition at $37{ }^{\circ} \mathrm{C}$. An objective lens HCX PL APO 40x/1.25-0.75 OIL was used except for Online Resource 2 and 7, for which HC PL APO 20x/0.70 IMM was used.

\section{Fluorescence microplate reader experiments}

\section{Culture}

MIN6 cells were seeded at a density of 6000 cells/well on 96-well clear-bottom plate ( $\mu$ Clear-plate \#655090, Greiner Bio-One). Wells in columns 3 and 5 (rows from B to F, total 12 wells) were used for culture and no cell was seeded in the top (A) and the bottom $(\mathrm{H})$ rows in these columns. Typically, $10 \mu \mathrm{l}$ of cell suspension was plated at $6 \times 10^{5}$ cells $/ \mathrm{ml}$ on the center of each well, and left for $20 \mathrm{~min}$ in $\mathrm{CO}_{2}$ incubator at $37^{\circ} \mathrm{C}$. $200 \mu$ of DMEM was then added to each well. Cells incubated for 10-14 DIV were used for measurement.

\section{Measurement}

For the measurement of the tracer uptake, a fluorescence microplate reader was used with its operation software (FlexStation and SoftMax Pro, Molecular Devices). The fluorescence was measured three times from the bottom of the plate and was averaged. Excitation, emission, and cutoff wavelength were 470, 540, and $495 \mathrm{~nm}$, respectively.

Just before measurements, culture medium was removed from each well leaving $50 \mu \mathrm{l}$. Cells were then washed five times with $200 \mu \mathrm{l}$ of standard Krebs-Ringer Buffer Solution (KRB) (in mM; $129 \mathrm{NaCl}, 4.8 \mathrm{KCl}, 1.2 \mathrm{KH}_{2} \mathrm{PO}_{4}, 1.2$ $\mathrm{MgSO}_{4}, 1.0 \mathrm{CaCl}_{2}, 10 \mathrm{HEPES}, 5.0 \mathrm{NaHCO}_{3}, 5.6 \mathrm{D}-\mathrm{glu}-$ cose, 0.1 carbenoxolone, $\mathrm{pH} 7.30-7.35)$ at room temperature $\left(26 \pm 1{ }^{\circ} \mathrm{C}\right)$. After the fifth wash, KRB was added to adjust the height of solution to that of blank wells in column 4 , in which $200 \mu \mathrm{l}$ of KRB without containing tracers was added. Nine regions of interest (ROIs, $1.5 \mathrm{~mm}$ in diameter, each contained typically $\sim 5000$ cells or more when measured at 12 DIV) were preset in single wells of 96-well plate. By visualizing cells before and after the experiment with a flatbed scanner (GT-X820, Seiko Epson), ROIs, in which cells were unevenly seeded or lost during washout, were excluded from the analysis.

Autofluorescence was measured for individual ROIs. According to a precisely timed protocol, $50 \mu$ of 2-NBDGor 2-NBDLG-containing KRB solution $(400 \mu \mathrm{M})$ was then added into 8 wells from $\mathrm{A}$ to $\mathrm{H}$ in column 3 , in which $50 \mu \mathrm{l}$ of KRB was pre-loaded, using a calibrated 8-channel pipette (final concentration, $200 \mu \mathrm{M}$ ). $30 \mathrm{~s}$ later, fluorescent tracers were similarly added to wells in column 5 . The top (A) and bottom $(\mathrm{H})$ wells in columns 3 and 5 were used as control to check that the tracer was successfully washed out from the solution. After adding the tracer solution, the plate was quickly placed on the tray in FlexStation at $37^{\circ} \mathrm{C}$. Five minutes later, $50 \mu \mathrm{l}$ of the tracer solution was removed from wells in column 3, and $300 \mu$ of KRB 
solution was added at room temperature. Thirty seconds later, similar washout process was done in column 5. After repeating this process 7 times, $300 \mu \mathrm{l}$ of KRB was finally added and $150 \mu \mathrm{l}$ of KRB was removed; the fluorescence was then measured and compared among ROIs. Transient increase in fluorescence due to a loss of membrane integrity decreased to a large extent during this washout procedure.

For $\mathrm{Na}^{+}$-free experiments, $\mathrm{NaCl}$ in $\mathrm{KRB}$ was replaced by choline chloride. For glucose competition assay, $50 \mathrm{mM}$ of D- or L-glucose solution was prepared by reducing $\mathrm{NaCl}$ so that the osmolality of the solution was identical to that of control.

\section{Reagents}

2-NBDLG (23003-v, Peptide Institute), 2-NBDG (23002-v, Peptide Institute), and 2-TRLG were provided by Peptide Institute Inc. and used as described previously [17]. Carbenoxolone (C4790, Sigma) was used to block gap junction/hemi-channel. Phloretin (P7912, Sigma), cytochalasin B (C6762, Sigma), and 4,6- $O$-ethylidene-D-glucose (E0402, Tokyo Chemical Industry) were applied prior to the tracer application. DAPI (D523, Wako) was added in some experiments for nuclear staining. pSIVA-IANBD (IMGENEX) and propidium iodide (IMGENEX) were used as a polarity-sensitive, real-time marker for apoptotic cells and an indicator for necrotic cells, respectively, according to the manufacturer's instruction. NBD- $\mathrm{NH}_{2}$ was synthesized by the reaction of NBD-F and ammonia. NBD$\mathrm{OH}$ was obtained as a by-product on the synthesis of 2-NBDLG. Both NBD-NH $\mathrm{N}_{2}$ and NBD-OH were used at a final concentration of $200 \mu \mathrm{M}$.

\section{Statistics}

ANOVA and Bonferroni-Dunn test were used. Error bars represent SD.

\section{Results}

\section{Imaging of 2-NBDG and 2-NBDLG uptake with confocal microscopy}

MIN6 cells, when seeded at a very low density (1000 cells per coverslip), formed small three-dimensional spheroid after several days (Fig. 1a, e). At 6 DIV, a brief
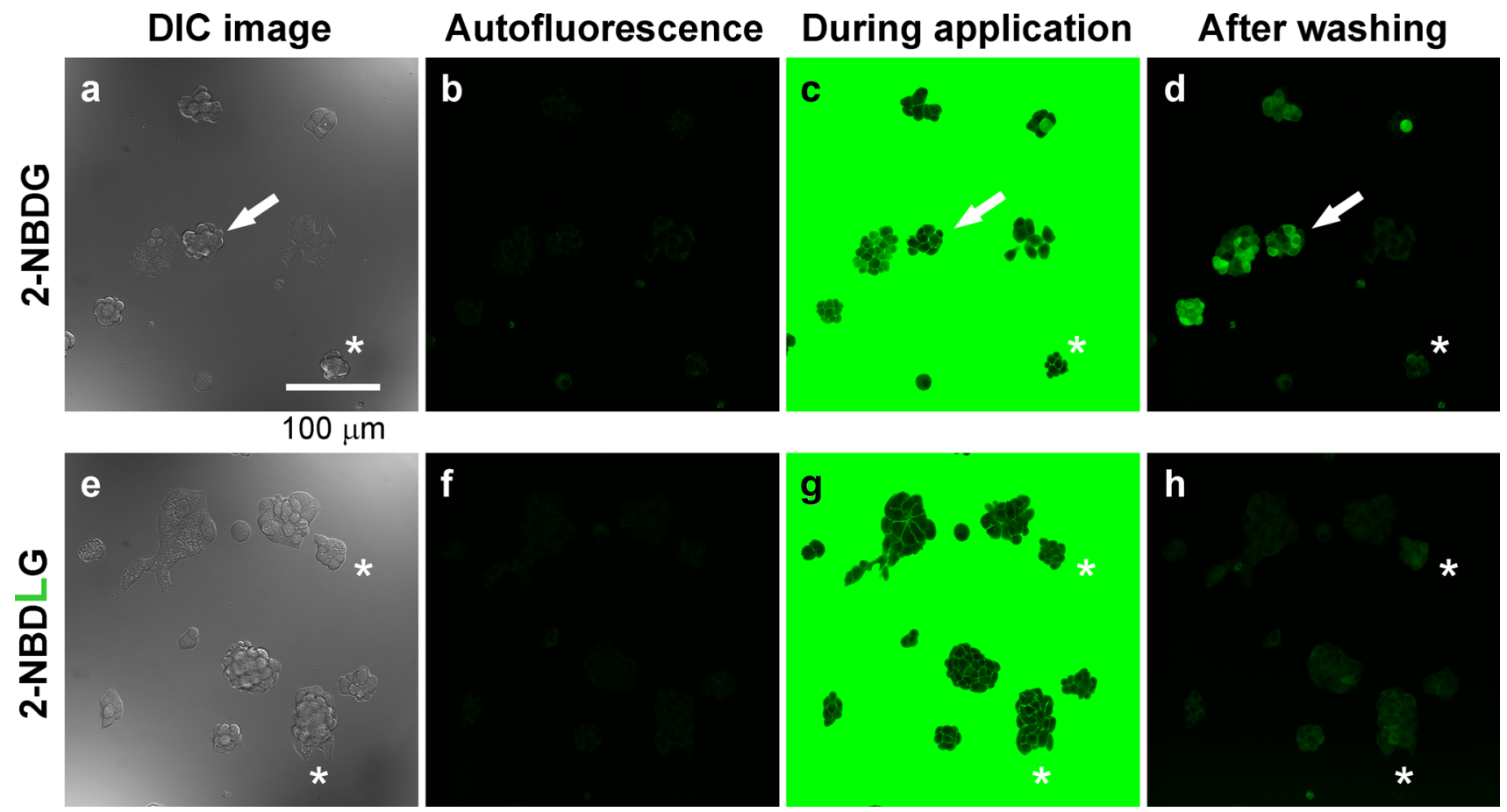

Fig. 1 Representative images of MIN6 cell spheroids subjected to a brief application of solution containing either 2-NBDG (a-d) or 2-NBDLG (e-h) at 6 days in vitro (DIV). a Differential interference contrast (DIC) image. b Autofluorescence before application of the fluorescent tracer. c During application of $100 \mu \mathrm{M}$ of 2-NBDGcontaining solution for $3 \mathrm{~min}$. d Fluorescence image taken at $4 \mathrm{~min}$ after starting washout of the tracer. 2-NBDG uptake varied considerably from prominent (arrow) to minimal (asterisk) among cells. e-h Similar to a-d but for application of $100 \mu \mathrm{M}$ of 2-NBDLG to the same series of culture examined on the same day. The uptake of 2-NBDLG into MIN6 cells at this stage was very weak, although some cells showed faint fluorescence (asterisks). Scale bar is common to all images 
superfusion with $100 \mu \mathrm{M}$ of 2-NBDG solution for $3 \mathrm{~min}$ followed by washout increased the cellular fluorescence in varying intensity among cells (Fig. 1b-d). Such unique heterogeneity in the 2-NBDG uptake is very different from relatively homogeneous uptake of the tracer in two-dimensionally spreading MIN6 cells (Online Resource 2). By contrast, no such increase in the fluorescence was detected by similarly applying $100 \mu \mathrm{M}$ of 2-NBDLG (L-form isomer) solution (Fig. 1e-h), although one may notice cells showing faint fluorescence if examined closely (asterisks in Fig. 1e-h).

Interestingly, more drastic 2-NBDLG uptake was detected at 10-15 DIV, when much thicker ( $>50 \mu \mathrm{m}$-thick) spheroids were grown (A in Fig. 2). Of two similarly shaped MIN6 spheroids (A and B in Fig. 2a), only upper one showed remarkable 2-NBDLG uptake (Fig. 2b, d), whereas lower one increased fluorescence only slightly.

Nuclear staining with DAPI in live-cell condition further revealed that the upper spheroid consisted of heterogeneous cells, including large cells which bear unusually large and strongly DAPI-positive nucleus as well as small cells having nucleus of ordinary size (Fig. 2e, f; see also Online Resource 4). By contrast, the lower spheroid consisted of cells bearing evenly sized small nuclei that were only weakly positive for DAPI (B in Fig. 2e, f).

It is noteworthy that 2-NBDLG was taken up not only into such large presumably multinucleated cells but also into small cells having nucleus of ordinary size (Fig. 2d, f). DAPI-positive, fibrous processes were seen in the upper spheroid, in addition (Online Resource 4) [20]. Similar fibrous processes appeared in other clusters that occasionally took up 2-NBDLG (asterisks in Fig. 2e and Online Resource 4).

\section{Quantitative evaluation of the 2-NBDG and 2- NBDLG uptake by a fluorescent microplate reader}

Thick and large spheroids easily collapsed sometimes in hours. Moreover, whether or not spheroids of interest would take up abundant 2-NBDLG was difficult to be expected prior to imaging. Thus, we evaluated the average fluorescence intensity of a large number of MIN6 cells subjected to 2-NBDLG compared with those subjected to 2-NBDG at 10-14 DIV using a fluorescent microplate reader.

When $200 \mu \mathrm{M}$ of 2-NBDG was applied for $5 \mathrm{~min}$, the average fluorescence intensity of ROIs increased from $1.6 \pm 0.4$ arbitrary unit (AU, autofluorescence) to $12.2 \pm 2.6 \mathrm{AU}(n=48, p<0.0001$, Fig. 3a). 2-NBDLG, applied simultaneously but to other wells, also noticeably increased the fluorescence from $1.7 \pm 0.5$ to $6.7 \pm 1.6 \mathrm{AU}$ $(n=51, p<0.0001$, Fig. 3a). Measurements were performed in quadruplicate and the ratio of the net increase in
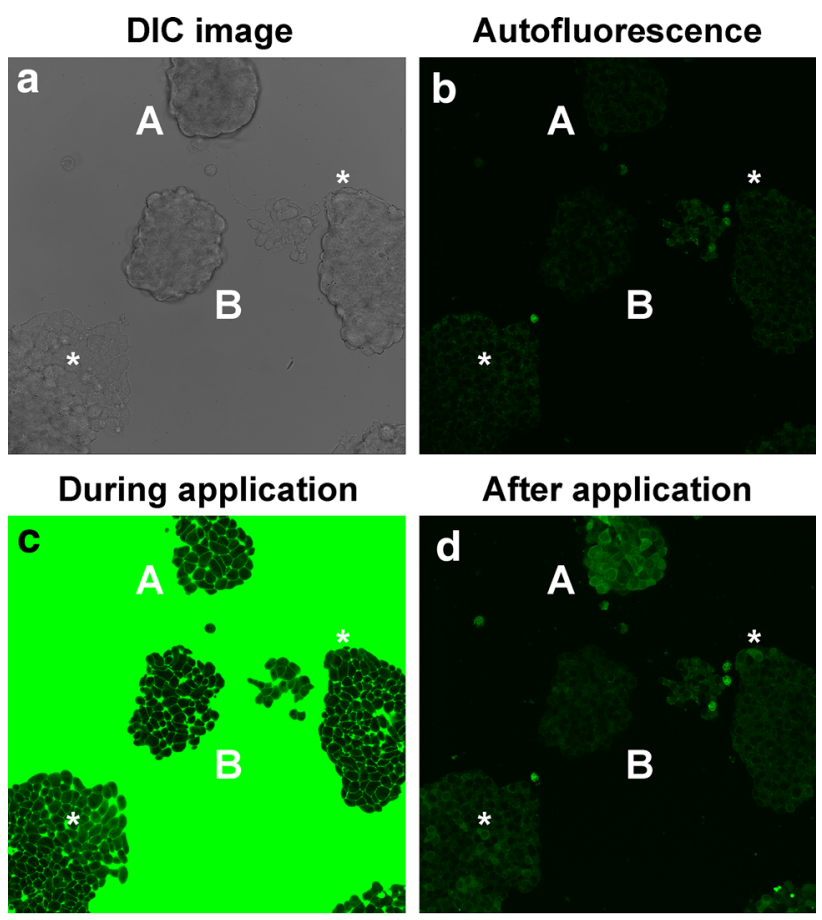

\section{After application}
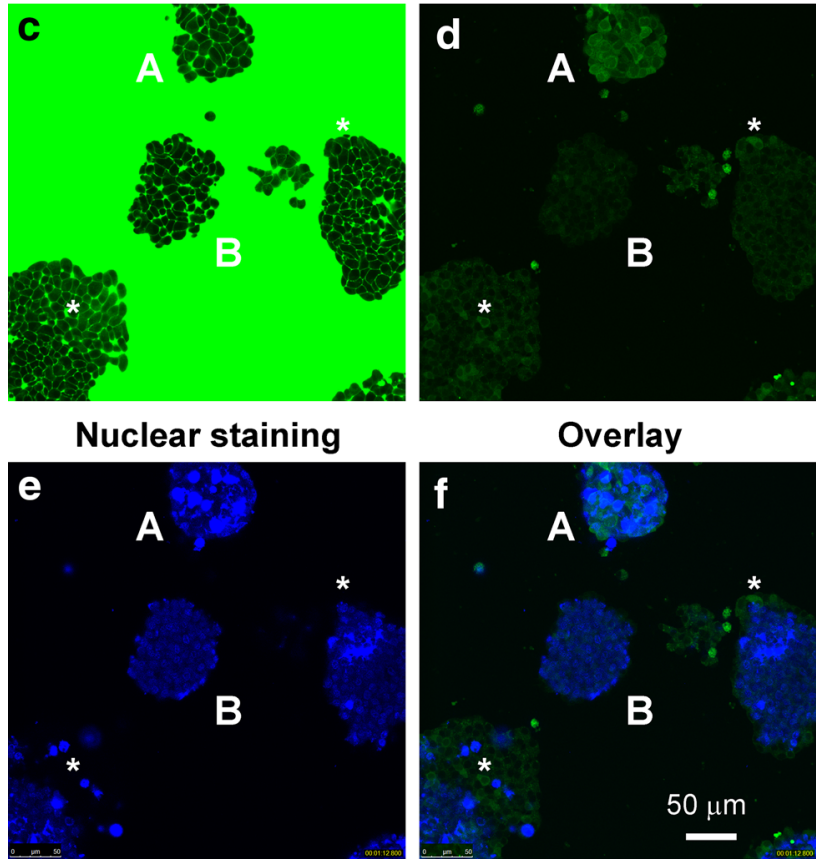

Fig. 2 Uptake of 2-NBDLG into MIN6 cells forming spheroids. a DIC image of MIN6 cells at 11 DIV. b Autofluorescence measured before application of $100 \mu \mathrm{M}$ of 2-NBDLG for $3 \mathrm{~min}$. c During application of 2-NBDLG. Note that both spheroids ( $A$ and $B$ ) were evenly superfused by 2 -NBDLG solution. d Two minutes after starting washout of the 2-NBDLG solution. Cells in the upper spheroid $(A)$ exhibited a strong 2-NBDLG fluorescence in the cytosol, whereas only a minimum increase in the fluorescence was seen in cells in the lower spheroid $(B)$. e Live-cell nuclear staining by DAPI, conducted after finishing 2-NBDLG application. Upper spheroid contained cells having extremely DAPI-positive large nucleus $(A)$, whereas lower one showed nuclei of normal size. $\mathbf{f}$ Merged image of d and e. Multi-stack z-sections of DAPI staining are available in Online Resource 4 in detail. Asterisks indicate cells moderately positive for 2-NBDLG in other spheroids. Scale bar is common to all panels

the fluorescence for 2-NBDLG to that for 2-NBDG was $44.9 \pm 1.7 \%$ in average (Fig. 3b).

Cytochalasin B, which acts as a GLUT inhibitor when used at a low dose $(10 \mu \mathrm{M})$, significantly decreased the 2-NBDG uptake into MIN6 cells $(p<0.0001$, Fig. 3c). 
Fig. 3 Quantitative evaluation of the 2-NBDG and 2-NBDLG uptake into MIN6 cells examined at 10-13 DIV. a Changes in the fluorescence of MIN6 cells subjected to 2-NBDG or 2-NBDLG solution. b The net increase in the fluorescence in (a). c Increase in the fluorescence in the absence or presence of a GLUT inhibitor cytochalasin B $(10 \mu \mathrm{M}, \mathrm{CB})$. d Effect of $\mathrm{Na}^{+}$-free condition on the 2-NBDG and 2-NBDLG (150 $\mu \mathrm{M}, \mathrm{PHT})$ on the 2-NBDG and 2-NBDLG uptake. Values are expressed as mean percent increase in the fluorescence relative to the fluorescence increase for 2-NBDG

application on the same 96-well plate (b-e). Values in individual columns represent mean fluorescence of more than 12 ROIs (more than 5000 cells are included in each ROI) and expressed as mean $\pm \mathrm{SD}$ uptake. e Effect of phloretin
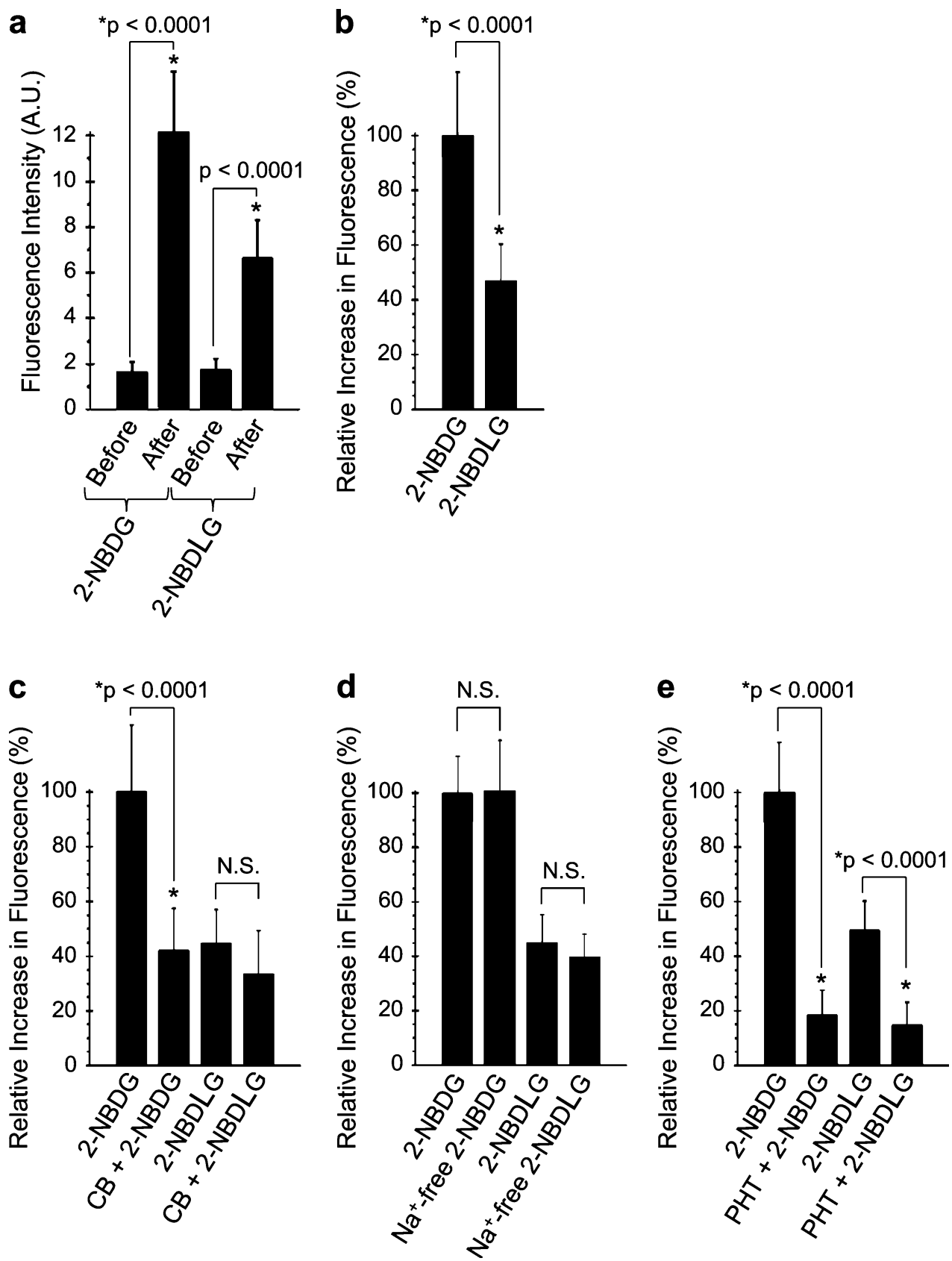

2-NBDG uptake was decreased by $61.9 \pm 3.9 \%$ on average in the presence of cytochalasin B in experiments performed in triplicate. On the other hand, 2-NBDLG uptake on the same plates was attenuated only slightly by $20.5 \pm 9.6 \%$ in the presence of cytochalasin B (Fig. 3c). Interpreted another way, in the presence of cytochalasin B, the substantial component remained in the uptake of 2-NBDLG, and that of 2-NBDG as well, implicating an involvement of non-GLUT mechanisms for both uptake of the L- as well as D-derivatives.

Consistently, a large amount of D-glucose $(50 \mathrm{mM})$ reduced the 2-NBDG uptake only moderately by
$28.9 \pm 12.4 \%(p<0.0001$, Online Resource 3a), and no inhibition was detected by the same amount of L-glucose. Similar to Fig. 3c, 2-NBDLG uptake was attenuated only slightly by $50 \mathrm{mM}$ D-glucose (Online Resource $3 b$ ). The same amount of L-glucose showed no effect on the uptake. An involvement of SGLTs, energy-demanding $\mathrm{Na}^{+}$-coupled glucose transporters, is unlikely, since both 2-NBDLG and 2-NBDG uptake persisted in the absence of $\mathrm{Na}^{+}$ion in the extracellular solution (Fig. 3d).

To our surprise, $150 \mu \mathrm{M}$ of phloretin, a broad spectrum inhibitor against membrane transport including GLUTs/ water channels [1,21], virtually abolished the increase in 
the fluorescence for 2-NBDLG as well as for 2-NBDG application, leaving only minimally detectable fluorescence $(p<0.0001$, Fig. 3e). Experiments were performed in quadruplicate and similar results were obtained. It is unlikely that the 2-NBDLG fluorescence was produced by fluorophore NBD, since the fluorescence of MIN6 cells was barely detectable when subjected to KRB containing either NBD-NH $\mathrm{N}_{2}$ or NBD-OH (data not shown).

\section{Cellular heterogeneity in spheroids revealed by a combined use of 2-NBDLG and 2-TRLG}

Tumor cells might internalize a wide variety of compounds if exposed for many minutes [22, 23]. To evaluate an occurrence of non-specific uptake, the second L-glucose derivative 2-TRLG [17] was applied simultaneously with 2-NBDLG (Fig. 4; Online Resource 1). 2-TRLG is a unique "membrane-impermeable" L-glucose derivative bearing Texas Red emitting red fluorescence [17]. As illustrated, combined administration of 2-NBDLG $(100 \mu \mathrm{M})$ and 2-TRLG $(20 \mu \mathrm{M})$ for 3 min to well-developed MIN6 spheroids (12 DIV) revealed spatially and temporally heterogeneous uptake among cells (Fig. 4). At 2 min after starting washout of the tracers, cells located in the central core of the spheroids turned yellow in merged image (Fig. 4e), indicating massive entrance of 2-NBDLG and 2-TRLG (Fig. 4b, c). However, most of these cells had lost the yellow color by 4 min after washout (Fig. 4k) due to a rapid exit of 2-NBDLG (Fig. 4h), while maintaining 2-TRLG intracellularly (Fig. 4i).

On the other hand, a considerable number of cells in the area surrounding the central core showed varied strength of green fluorescence, which well persisted at $4 \mathrm{~min}$ after washout (Fig. 4b, h, see Online Resource 5). The spatial distribution of such green cells largely overlapped with the area containing cells bearing only weakly DAPI-positive nuclei (Fig. 4a, g, f and 1), and with non-apoptotic/nonnecrotic region (Online Resource 7), suggesting that such 2-NBDLG-positive/2-TRLG-negative cells represent viable cells.

Cells showing pale red, deep red, and yellow color were distinguished in the surrounding area at $2 \mathrm{~min}$ (Fig. 4e). These fluorescent colors can be used as a measure reflecting loss of membrane integrity from severe to less severe in this order and, indeed, some deep red cells lost their color at $4 \mathrm{~min}$. Dark quiescent cells were found in peri-central region, which took up little 2-NBDLG as if they were normal cells (Fig. 4e, k). Similar cells could be found as well when D-glucose derivative 2-NBDG was applied similarly with 2-TRLG, appeared less prominent though (Online Resource 6). Further quantification of such dark cells was not conducted in the present study, because
Fig. 4 Confocal microscopic images of 12 DIV MIN6 spheroids subjected to $100 \mu \mathrm{M}$ of 2-NBDLG (green) and $20 \mu \mathrm{M}$ of 2-TRLG (red) mixture for $3 \mathrm{~min}$ followed by washout. a Nuclear staining with DAPI in live cell condition. The central core region of spheroids appears to be necrotic (see also d). b and $\mathbf{c}$ Fluorescence images taken at 2 min after starting washout of the tracers in the green (b, $500-580 \mathrm{~nm})$ and the red (c, 580-740 nm) channel, reflecting entrance of 2-NBDLG and 2-TRLG, respectively. d Differential interference contrast (DIC) image. e Overlay of the green, red, and DIC images. f Overlay of (a) and (e). Cellular heterogeneity is clearly seen by a combination of the two fluorescent colors. Cells indicated by arrows exhibited yellow color at $2 \mathrm{~min}(\mathbf{e})$, turned red at $4 \mathrm{~min}(\mathbf{k})$. This is because green 2-NBDLG was lost $(\mathbf{b}, \mathbf{h})$, while red 2-TRLG remained (c, i). If one saw a single 2-NBDLG image (b), cells indicated by arrows would have been misinterpreted to be similar to cells nearby. g-l Similar to a-f but images taken at 4 min after starting washout. Numbers of green cells with no red fluorescence, seen in the area surrounding the central core, preserved their color for at least up to $30 \mathrm{~min}(\mathbf{h}, \mathbf{i}, \mathbf{k})$. Also noted are dark cells in the area just surrounding the central core $(\mathbf{b}, \mathbf{e}, \mathbf{h}, \mathbf{k})$. Bars are common to all panels

only a small portion of tight and thick MIN6 spheroids demonstrated such typical profile of uptake.

\section{Discussion}

In the present study, we have shown that a small portion of insulin-secreting clonal cells (MIN6) took up abundant 2-NBDLG when they formed three-dimensional, multicellular spheroids exhibiting heterogeneous nuclei at a late culture stage. The 2-NBDLG uptake occurred specifically in a phloretin-inhibitable manner. A combined use of 2-NBDLG with a membrane-impermeable, red fluorescence-emitting L-glucose derivative 2-TRLG provides a unique method for visualizing heterogeneity of tumor cells in multiple colors.

\section{Characterization of tumor cells by fluorescent L-glucose derivatives}

Noticeable uptake of 2-NBDLG proceeded along with formation of three-dimensional spheroids, consisting of cells bearing nuclei of heterogeneous size accompanied by DAPI-positive fibrous processes in some cases. Such features are among major cytological criteria in clinical diagnosis for tumor cells suspected of high grade of malignancy.

Multi-cellular spheroids are thought to emulate the cellular heterogeneity in tumor typically found in the body cavity fluid and in solid tumors [24, 25]. In such threedimensionally grown tumor, there are cells in intermediate states between perfectly healthy and totally collapsed, due to insufficient oxygen/fuel supply and metabolite 


\section{2 minutes after washout}

a

d

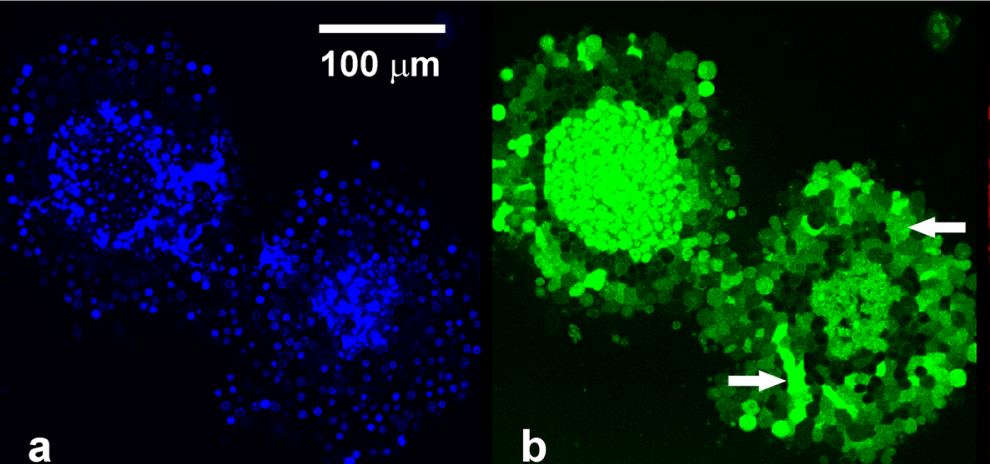

C
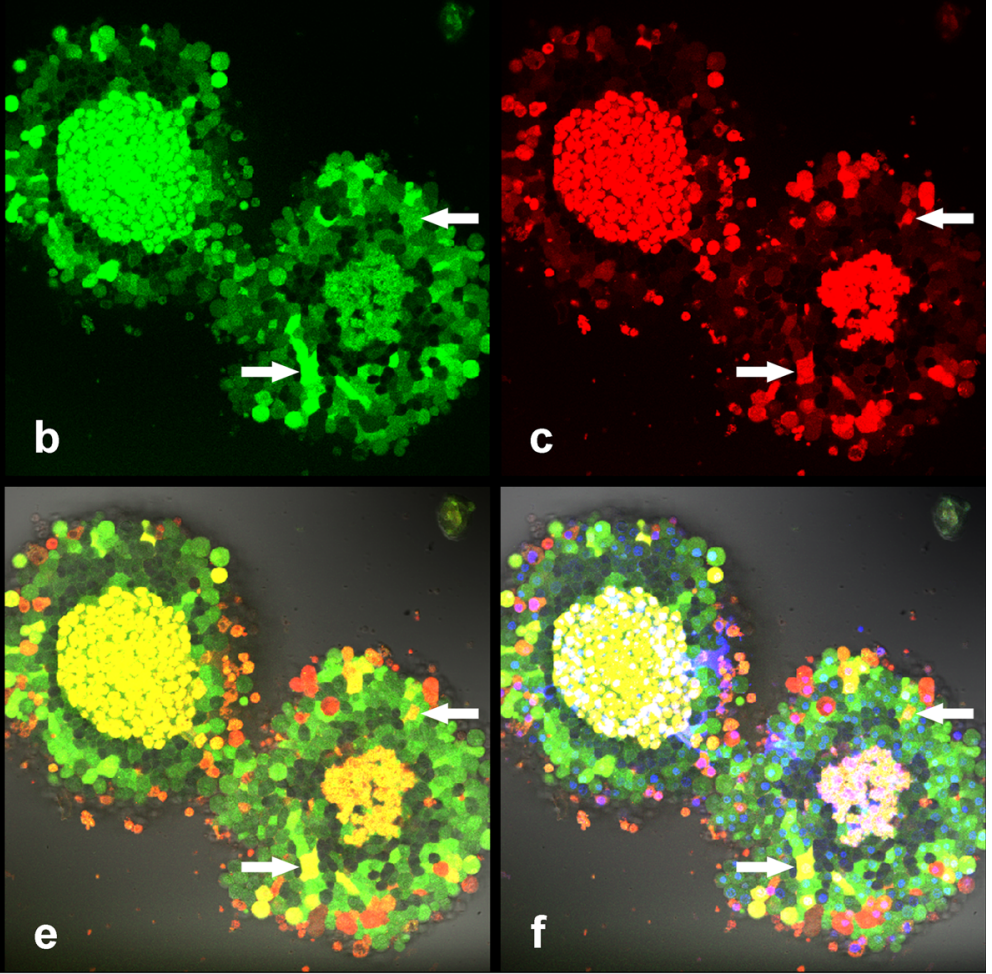

4 minutes after washout

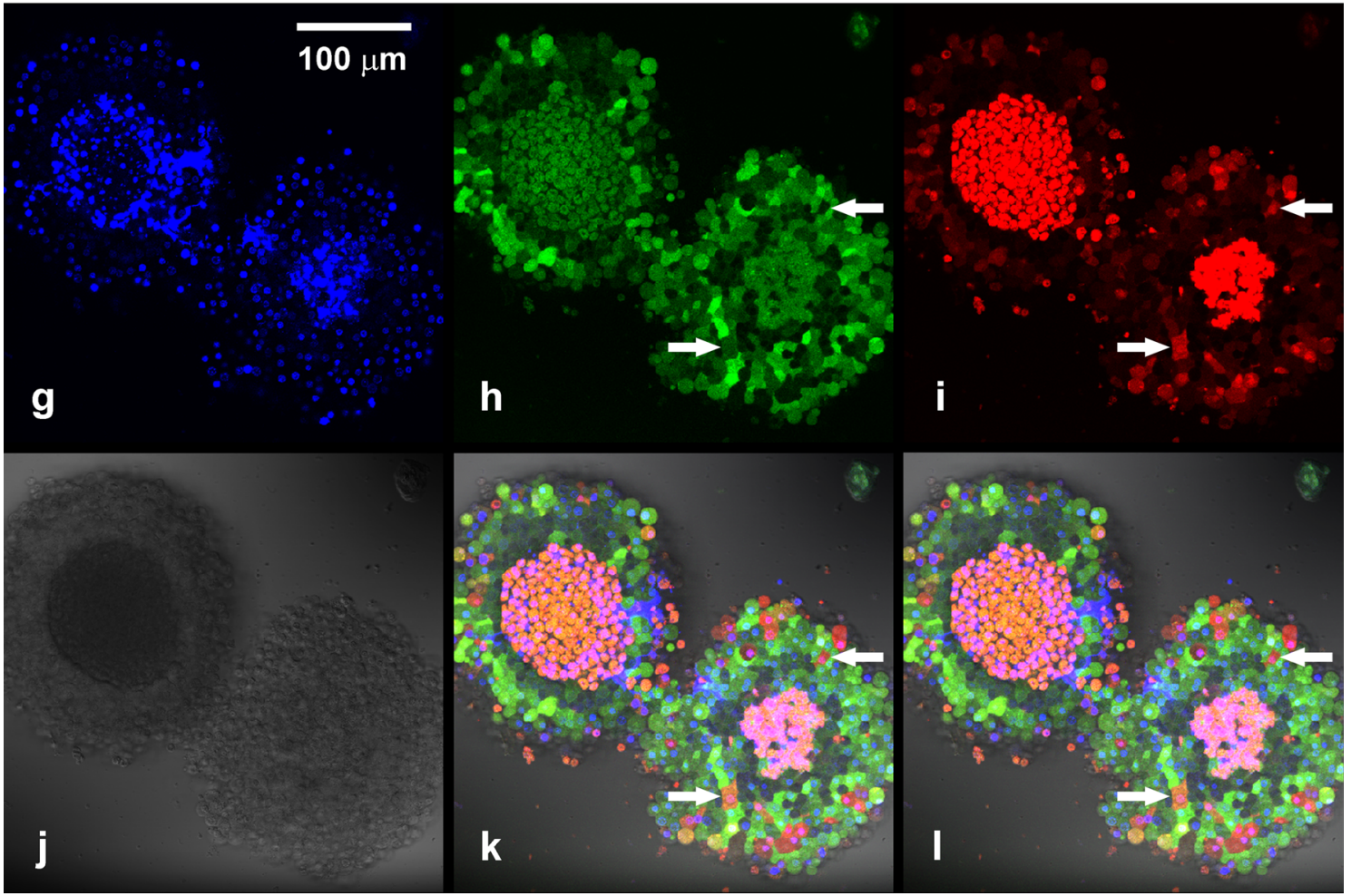


clearance, pharmacological treatment, and inflammatory response. The existence of such intermediate cells makes it often difficult to characterize tumor cells using functional probes such as 2-NBDG/2-NBDLG.

2-TRLG can be used as a sensitive measure for the membrane state. Once 2-TRLG entered into the cytosol of such intermediate cells, it remained intracellularly for tens of minutes, whereas it disappeared soon when permeated into totally collapsed cells (Fig. 4). Since a more hydrophilic analog of 2-TRLG failed to show such characteristics (data not shown), charged lipophilic property of 2-TRLG might well be related to the retention. Such unique feature of 2-TRLG is useful for recognizing partially damaged cells during live imaging, contrasted to a commercially available dead cell marker such as propidium iodide, which irreversibly binds to the nucleus once entering into cells (Online Resource 7).

Interestingly, there were cells sustaining 2-NBDLG fluorescence for over $30 \mathrm{~min}$ as well as dark and quiescent cells in the area surrounding the necrotic central core of spheroids. Questions are posed what the difference between 2-NBDLG-positive and negative cells is, and whether 2-NBDLG is metabolized after entering into tumor cells.

\section{Mechanistic consideration underlying 2-NBDLG uptake into tumor cells}

From the fact that neither cytochalasin B nor a large amount of D-glucose could totally abolish the uptake of 2-NBDLG and of 2-NBDG, it may be speculated that MIN6 insulinoma expresses phloretin-sensitive, but nonGLUT, non-stereoselective pathways when forming multicellular spheroids. Glucose entry into cells might well occur not only through saturable (carrier-mediated) processes requiring glucose binding to the postulated recognition site of the transporter like GLUTs [26, 27] but also through non-saturable ones. Indeed, use of 2-NBDG has suggested in some plant cells that a mercury-sensitive, water-channel-like mechanisms operate concomitantly with saturable processes [28]. 2-NBDLG uptake into MIN6 cells was virtually abolished by phloretin, which is the aglycone of phlorizin, a phytoalexin produced by such as apple and cherry trees. Phloretin, being used as a GLUT inhibitor for many years, is also known as a potent inhibitor of aquaporins [21]. Thus, it is of interest to examine if such channel-like processes participate in the glucose transport in insulinoma cells [21, 29].

A large amount of 4,6- $O$-ethylidene-D-glucose has been used by some investigators as a competitive inhibitor against GLUT-mediated 2-NBDG transport, postulating that it selectively interacts with the outward-facing sugar binding site of a carrier [9]. However, 4,6-O-ethylidene-D- glucose might not fully reflect the stereoselective property of D-glucose, since its mirror-image isomer also significantly inhibited 2-NBDG uptake into MIN6 cells (data not shown).

\section{Clinical significance of the uptake of fluorescent L-glucose derivatives}

Evidence has been accumulated which shows that 2-NBDG is taken up into variety of tumor cell lines, such as derived from melanoma, breast, liver, colorectal cancers, and glioma $[10,12,22,27]$. 2-NBDG has also been used as a contrast agent for imaging biopsy specimen obtained from oral, breast, and Barrett's esophagus cancer patients [11, 13, 14]. However, critical issues to be solved when using D-glucose derivatives in tumor imaging may include how to reduce the uptake into normal tissues containing fat and muscle, and how to discriminate the uptake into tumor cells from that due to inflammatory and/or non-cancerous anomalies $[12,14]$. Difficulty in applying them for diabetic patients may also be pointed out. The least interaction with GLUTs, if any, of Lglucose derivatives like 2-NBDLG would make undesirable binding to non-tumor cells, or toxic effect in other words, minimum compared to that expected when using 2-NBDG.

One of the key therapeutic strategies against rapidly growing tumor would be deprivation of energy source and nutrients [30]. However, tumor cells that survive in lownutrient environment might well develop regulatory mechanisms enabling utilization of unusual carbohydrate as a carbon source as reported in some lower organisms $[31,32]$. Heterogeneous nuclei and tubulin-like fibrous structure are among important features of malignant tumor spheroids [33]. Illuminating the breakdown in the stereoselectivity of tumor cells by their heterogeneous acceptance of unnatural sugar/sugar analogs might serve a new indication for cancer diagnosis eventually at the single cell level and help determining therapies against cancer. Further investigation is required to clarify the transport mechanism, intracellular fate, as well as limitation of 2-NBDLG in tumor imaging.

Acknowledgments This research was supported by Science and Technology Incubation Program in Advanced Regions (KY, TY), Support for Increasing the Value for University Patents (KY), Collaborative Research Based on Industrial Demand (KY), and A-STEP (TY, TT, KY) from JST, Grant-in-Aid for Scientific Research on Priority Areas (KY, 20019003, 20056001), Research Funds from Research Foundation for Opto-Science and Technology (KY), and Grant for Hirosaki University Institutional Research (KY). A part of the present study was seen on a patent pending (WO2012/133688). The authors thank Drs. Junichi Miyazaki (Osaka Univ.) for providing us MIN6 cells, Sechiko Suga, Seiji Watanabe and Kazuyoshi Hirota (Hirosaki Univ.) for fluorescence measurement, Hideaki Matsuoka (Tokyo Univ. Agri. Technol.), Hirotaka Onoe and Tsuyoshi Tahara (Riken), Iwao Kanno, Kazuto Masamoto, and Yukie Yoshii (National 
Inst. Radiol. Sci.) for helpful discussion, Masahito Kogawa and Rumiko Narita (Hirosaki Univ.) for technical assistance.

\section{Compliance with ethical standards}

Conflict of interest KY, TY, and TT received grants above noted from the Japanese government for developing potential cancer diagnostic agents, in which $\mathrm{AS}, \mathrm{KN}, \mathrm{KO}$ and $\mathrm{YO}$ are collaborators. KY, TY and TT, and in one case AS and YO, are co-applicants for multiple patents related to cancer diagnostics using fluorescent L-glucose derivatives. KY, AS, and $\mathrm{KO}$ assigned ownership of all these patents to Hirosaki University.

Open Access This article is distributed under the terms of the Creative Commons Attribution 4.0 International License (http://creative commons.org/licenses/by/4.0/), which permits unrestricted use, distribution, and reproduction in any medium, provided you give appropriate credit to the original author(s) and the source, provide a link to the Creative Commons license, and indicate if changes were made.

\section{References}

1. Zhao FQ, Keating AF. Functional properties and genomics of glucose transporters. Curr Genom. 2007;8:113-28.

2. Yoshioka K, Takahashi H, Homma T, et al. A novel fluorescent derivative of glucose applicable to the assessment of glucose uptake activity of Escherichia coli. Biochim Biophys Acta. 1996;1289:5-9.

3. Yamada K, Nakata M, Horimoto N, Saito M, Matsuoka H, Inagaki N. Measurement of glucose uptake and intracellular calcium concentration in single, living pancreatic beta-cells. J Biol Chem. 2000;275:22278-83.

4. Yamada K, Saito M, Matsuoka H, Inagaki N. A real-time method of imaging glucose uptake in single, living mammalian cells. Nat Protoc. 2007;2:753-62.

5. Ohtsubo K, Takamatsu S, Minowa MT, Yoshida A, Takeuchi M, Marth JD. Dietary and genetic control of glucose transporter 2 glycosylation promotes insulin secretion in suppressing diabetes. Cell. 2005;123:1307-21.

6. Bernardinelli Y, Magistretti PJ, Chatton JY. Astrocytes generate $\mathrm{Na}+$-mediated metabolic waves. Proc Natl Acad Sci USA. 2004;101:14937-42.

7. Rouach N, Koulakoff A, Abudara V, Willecke K, Giaume C. Astroglial metabolic networks sustain hippocampal synaptic transmission. Science. 2008;322:1551-5.

8. Gandhi GK, Cruz NF, Ball KK, Theus SA, Dienel GA. Selective astrocytic gap junctional trafficking of molecules involved in the glycolytic pathway: impact on cellular brain imaging. J Neurochem. 2009;110:857-69.

9. Barros LF, Courjaret R, Jakoby P, Loaiza A, Lohr C, Deitmer JW. Preferential transport and metabolism of glucose in Bergmann glia over Purkinje cells: a multiphoton study of cerebellar slices. Glia. 2009;57:962-70.

10. O'Neil RG, Wu L, Mullani N. Uptake of a fluorescent deoxyglucose analog (2-NBDG) in tumor cells. Mol Imaging Biol. 2005;7:388-92.

11. Nitin N, Carlson AL, Muldoon T, El-Naggar AK, Gillenwater A, Richards-Kortum R. Molecular imaging of glucose uptake in oral neoplasia following topical application of fluorescently labeled deoxy-glucose. Int J Cancer. 2009;124:2634-42.

12. Sheth RA, Josephson L, Mahmood U. Evaluation and clinically relevant applications of a fluorescent imaging analog to fluorodeoxyglucose positron emission tomography. J Biomed Opt. 2009;14:064014.
13. Langsner RJ, Middleton LP, Sun J, et al. Wide-field imaging of fluorescent deoxy-glucose in ex vivo malignant and normal breast tissue. Biomed Opt Express. 2011;2:1514-23.

14. Thekkek N, Maru DM, Polydorides AD, Bhutani MS, Anandasabapathy S, Richards-Kortum R. Pre-clinical evaluation of fluorescent deoxyglucose as a topical contrast agent for the detection of Barrett's-associated neoplasia during confocal imaging. Technol Cancer Res Treat. 2011;10:431-41.

15. Viale A, Pettazzoni P, Lyssiotis CA, et al. Oncogene ablationresistant pancreatic cancer cells depend on mitochondrial function. Nature. 2014;514:628-32.

16. Yamamoto T, Nishiuchi Y, Teshima T, Matsuoka H, Yamada K. Synthesis of 2-NBDLG, a fluorescent derivative of L-glucosamine; the antipode of D-glucose tracer 2-NBDG. Tetrahedron Lett. 2008;49:6876-8.

17. Yamamoto T, Tanaka S, Suga S, et al. Syntheses of 2-NBDG analogues for monitoring stereoselective uptake of D-glucose. Bioorg Med Chem Lett. 2011;21:4088-96.

18. Miyazaki J, Araki K, Yamato E, et al. Establishment of a pancreatic beta cell line that retains glucose-inducible insulin secretion: special reference to expression of glucose transporter isoforms. Endocrinology. 1990;127:126-32.

19. O'Driscoll L, Gammell P, McKiernan E, et al. Phenotypic and global gene expression profile changes between low passage and high passage MIN-6 cells. J Endocrinol. 2006;191:665-76.

20. Bonne D, Heusele C, Simon C, Pantaloni D. 4',6-Diamidino-2phenylindole, a fluorescent probe for tubulin and microtubules. J Biol Chem. 1985;260:2819-25.

21. Tsukaguchi H, Shayakul C, Berger UV, et al. Molecular characterization of a broad selectivity neutral solute channel. J Biol Chem. 1998;273:24737-43.

22. Cheng Z, Levi J, Xiong Z, et al. Near-infrared fluorescent deoxyglucose analogue for tumor optical imaging in cell culture and living mice. Bioconjug Chem. 2006;17:662-9.

23. Kelf TA, Sreenivasan VK, Sun J, Kim EJ, Goldys EM, Zvyagin AV. Non-specific cellular uptake of surface-functionalized quantum dots. Nanotechnology. 2010;21:285105.

24. Pampaloni F, Reynaud EG, Stelzer EH. The third dimension bridges the gap between cell culture and live tissue. Nat Rev Mol Cell Biol. 2007;8:839-45.

25. Elliott NT, Yuan F. A review of three-dimensional in vitro tissue models for drug discovery and transport studies. J Pharm Sci. 2011;100:59-74.

26. Lefevre PG. Sugar transport in the red blood cell: structure-activity relationships in substrates and antagonists. Pharmacol Rev. 1961;13:39-70.

27. Deng D, Xu C, Sun P, et al. Crystal structure of the human glucose transporter GLUT1. Nature. 2014;510:121-5.

28. Conde C, Silva P, Agasse A, Tavares RM, Delrot S, Geros H. An Hgsensitive channel mediates the diffusional component of glucose transport in olive cells. Biochim Biophys Acta. 2007;1768:2801-11.

29. Delporte C, Virreira M, Crutzen R, et al. Functional role of aquaglyceroporin 7 expression in the pancreatic beta-cell line BRIN-BD11. J Cell Physiol. 2009;221:424-9.

30. Cairns RA, Harris IS, Mak TW. Regulation of cancer cell metabolism. Nat Rev Cancer. 2011;11:85-95.

31. Sasajima KI, Sinskey AJ. Oxidation of L-glucose by a pseudomonad. Biochim Biophys Acta. 1979;571:120-6.

32. Shimizu T, Takaya N, Nakamura A. An L-glucose catabolic pathway in Paracoccus species 43P. J Biol Chem. 2012;287:40448-56.

33. McCarroll JA, Gan PP, Erlich RB, et al. TUBB3/betaIII-tubulin acts through the PTEN/AKT signaling axis to promote tumorigenesis and anoikis resistance in non-small cell lung cancer. Cancer Res. 2015;75:415-25. 